\title{
Stochastic Programming Models and Hybrid Intelligent Algorithm for Unbalanced Bidding Problem
}

\author{
Xingzi Liu \\ Department of Maths and Physics \\ Guilin University of technology \\ Guilin 541004, China
}

Tel: 86-773-589-9437 E-mail: angelxingzi@yahoo.com.cn

Liang Lin

Department of Maths and Physics

Guilin University of technology

Guilin 541004, China

Tel: 86-773-589-7053Ｅ-mail: linliang6666@126.com

Dongran Zang

Department of Maths and Physics

Guilin University of technology

Guilin 541004, China

Tel: 86-773-589-9437Ｅ-mail: zangdr@163.com

The research is financed by Guangxi Natural Science Foundation of China(GuiNo. 0833621) and Innovation Project of Guangxi Graduate Education(No. 2008105960202M29)

\begin{abstract}
The expected value model and the chance-constrained programming model for unbalanced bidding problem are established on the condition that quantities of each activity are stochastic variables and the total project is finished smoothly in this paper. These models can make the unbalanced bidding price more reasonable and applicable. In order to solve these models, stochastic simulation, neural network and genetic algorithm are integrated to produce a hybrid intelligent algorithm. Finally, a numerical example is given to illustrate its effectiveness.
\end{abstract}

Keywords: Unbalanced bidding, Stochastic variable, Stochastic programming, Hybrid intelligent algorithm

\section{Introduction}

With the further development of the bid mechanism, the engineering item invitation has already become more and more standardized. How to master the strategy of invitation, increaseing the efficiency of bids is very important for the bidders. In order to make the bid price more competitive and obtain sufficient profits in engineering bids, they can use many kinds of methods to calculate the bid price, including unbalanced bidding, unexpected markdown, loss-first-profit-later quotation, multiple alternative quotation, etc. On the whole, unbalanced bidding is the most widely used tactic.

Unbalanced bids is opposite to the conventional balanced bids, which is a wide-used method in the optimization of unit 
price internationally. It can be described as follows: after the contractors carry on the resource distribution, the cost analysis and the research of bidding skills according to bills of quantities provided by the owners, they enhance some comprehensive unit price in bills of quantities consciously and reduce other comprehensive unit price simultaneously in order to obtain more economic benefits without raising the total price.

Unbalanced bids is divided into two types of "earlier receiving money" and "more receiving money". However, how to realize these two types lacks quantitative optimization models and efficient algorithms, so it can bring randomness and blindness in process of bidding. In order to solve this problem, many researchers at home or abroad have carried on careful exploration and research. Dayanand and Padman(1997, p.906 \& 2001, p.197) initially established optimal models of the project payment scheduling problem from contractor's and client's viewpoints respectively, also presented a heuristic algorithm to find the payment schedule of maximizing project profits. Afterwards Ulusoy et al. (2000, p.262) introduced a method of seeking the equal payment scheduling from both sides of contractor and client. In our country, the research of this problem is still on the beginning period, and most researches were presented theoretically. $\mathrm{Xu}(1990)$ 's research result in the field of project cost management has laid a solid theoretical foundation for other researchers; After that, Zhang et al. (2005, p.595) studied bids strategy via the game theory, using the characteristics of engineering quality list in the market economy. Chen et al. $(2005, \mathrm{p} .118)$ built linear programming models with objective of maximizing surplus profits according to the fundamental principles and the conditions of unbalanced bids; $\mathrm{He}$ and $\mathrm{Xu}(2007, \mathrm{p} .474)$ set up nonlinear mixed integer programming models from contractor's and client's viewpoints respectively, also designed the two-module simulated annealing heuristics algorithm to solve these models. Generally speaking, most of these researches were built in certain environments, and the results showed that it could be optimized to give the best present value of actual payment without raising the total price when the unit price of front activities were enhanced by $10 \%$ and the unit prices of following activities were reduced by $10 \%$.

In the real-world situation, unbalanced bids is a complex problem. If contractors overuse unbalaned unit price, it can not only bring the loss to contractors or influence winning a bid, but also result in the serious trouble and increase the investment risk to clients during the project management process. Therefore, there is a need to study unbalanced bidding problem in uncertain environments so as to make the contractor's bid price more competitive and improve the practical application value of unbalanced bids. Due to the budget engineering quantities uncertainty and substitution of main components in each activity, engineering quantities of each activity are stochastic variables within certain limits.

In this paper, we will discuss unbalanced bidding problem with stochastic bidding engineering quantities based on operations research and uncertain programming theory. First, the expected value model and the chance-constrained programming model with objective of maximizing the present value of actual payment without raising the total price for unbalanced bids will be established respectively on the condition that the bidding engineering quantities of each activity are stochastic variables and the total project is finished smoothly. Then stochastic simulation, neural network and genetic algorithm will be integrated to produce a hybrid intelligent algorithm for solving these models. Finally, a numerical example will be given to illustrate the effectiveness of the algorithm. The numerical result is successful, which can make the unbalanced bidding price more reasonable and applicable.

\section{Problem Description}

Before we begin to study unbalanced bidding problem with stochastic bidding engineering quantities, we first make some assumptions as:

(a) the contractor's anticipated starting time and duration time of each activity are the same as owner's;

(b) the interest rate doesn't change during the period of the project;

(c)each activity should be processed without interruption;

(d)the owner decides payment according to the construction schedule of each activity;

(e) the last paymen must be arranged when the total project is finished.

For simplicity, we assume that bill of quantity(BOQ) consists of $n$ bidding activities, and the ith bidding activities have $m$ main components, $i=1,2, \cdots, n$, respectively.

Next, in order to model unbalanced bids problem, we must introduce the following indices and parameters:

$t_{s i}:$ the starting time of the ith activities;

$t_{i}:$ the duration time of the ith activities;

$r:$ the interest rate;

$k_{i}$ : the discounting coefficient of construction cost needed for the ith bidding activities, and it can be calculated by 
the following equation, $k_{i}=\frac{(1+r)^{t_{i}}-1}{t_{i} \cdot r \cdot(1+r)^{t_{s i}+t_{i}}}$

$P_{i}$ : the budget price for the ith activities;

$\bar{P}_{i}$ : the bidding price for the ith activities;

$q_{i j}$ : the budget engineering quantities for the $j t h$ components in the ith activities, $j=1,2, \cdots, m$;

$\xi_{i j}$ : the uncertain bidding engineering quantities for the $j t h$ components in the ithactivities;

$p_{i j}$ : the budget unit price of the client for the $j t h$ components in the ithactivities;

$x_{i j}$ : the bidding unit price of the contractor for the $j t h$ components in the ith activities;

According to the assumptions, the budget price of the client for the total project should be $P=\sum_{i=1}^{n} P_{i}$.

The bidding price of the contractor for the total project is $\bar{P}=\sum_{i=1}^{n} \bar{P}_{i}$.

The budget engineering quantities of the client for the ith activities should be $q_{i}=\sum_{j=1}^{m} q_{i j}$.

The uncertain bidding engineering quantities of the contractor for the ith activities should be $\xi_{i}=\sum_{j=1}^{m} \xi_{i j}$.

The budget price for the total project is $P_{i}=\sum_{j=1}^{m} q_{i j} p_{i j}$.

The bidding price for the total project is $\overline{P_{i}}=\sum_{j=1}^{m} \xi_{i j} x_{i j}$.

Therefore, the present value of the client's budget price for the total project can be written as $f=\sum_{i=1}^{n} k_{i} P_{i}$.

The present value of the contractor's bidding price for the total project can be written as $\bar{f}=\sum_{i=1}^{n} k_{i} \bar{P}_{i}$.

As these parameters and basic formulas have been given in the above section, we can establish different stochastic programming models to satisfy different goals.

\section{Stochastic models of unbalanced bidding problem}

\subsection{Expected value model}

The first type of stochastic programming is the expected value model(EVM), which optimizes the expected objective function subject to a set of expected constraints. The expected value model has been applied in a wide variety of real-world problems. In practice, the bidding engineering quantity $\xi_{i j}$ is usually a stochastic variable, so is the present value function $\bar{f}$ of contractor's bidding price for the total project. Since we can't predict the present value of contractor's bidding price for the total project accurately, a natural idea is to employ the expected present value $E[\bar{f}]$. Because we want to find the decision with maximum expected return subject to some expected constraints, then we have the following EVM: 


$$
\max E[\bar{f}]=E\left[\sum_{i=1}^{n} k_{i} \overline{P_{i}}\right]
$$

subject to:

$$
\begin{aligned}
& E\left[\sum_{i=1}^{n} \overline{P_{i}}-\sum_{i=1}^{n} P_{i}\right] \leq 0 \\
& E\left[\sum_{i=1}^{n} \sum_{j=1}^{m} \xi_{i j}-\sum_{i=1}^{n} \sum_{j=1}^{m} q_{i j}\right] \leq 0 \\
& 0.9 p_{i j} \leq x_{i j} \leq 1.1 p_{i j}, \quad i=1,2, \cdots, n ; j=1,2, \cdots, m .
\end{aligned}
$$

where $x_{i j}$ is the decision variable, $\xi_{i j}$ is the stochastic variable, the form (2) and the form (3) express expected constraints, the form (4) expresses the limits of contractor's bidding unit price in order to avoid suspicion of unbalanced bidding according to many practical cases.

\subsection{Chance-constrained model}

The second type of stochastic programming is chance-constrained programming (CCP), which was initialized by Charnes and Cooper(1959, p.73). Its outstanding feature characteristic is that the stochastic constraints will hold at least some given confidence levels. For the same reason, we assume that $x_{i j}$ is the decision variable, $\xi_{i j}$ is the stochastic variable, and the present value function $\bar{f}$ of the contractor's bidding price for the total project is the return function. Then in order to maximize the present value of the contractor's bidding price with a given confidence level subject to some chance constraints, we build the following chance-constrained model based on stochastic CCP:

$\max \bar{f}$

subject to:

$$
\begin{aligned}
& \operatorname{Pr}\left\{\sum_{i=1}^{n} k_{i} \overline{P_{i}} \geq \bar{f}\right\} \geq \alpha \\
& \operatorname{Pr}\left\{\sum_{i=1}^{n} \bar{P}_{i} \leq \sum_{i=1}^{n} P_{i}\right\} \geq \beta \\
& \operatorname{Pr}\left\{\sum_{i=1}^{n} \sum_{j=1}^{m} \xi_{i j} \leq \sum_{i=1}^{n} \sum_{j=1}^{m} q_{i j}\right\} \geq \gamma \\
& 0.9 p_{i j} \leq x_{i j} \leq 1.1 p_{i j}, \quad i=1,2, \cdots, n ; j=1,2, \cdots, m .
\end{aligned}
$$

where $\alpha, \beta, \gamma$ are the predetermined confidence levels, the form (5), the form (6) and the form (7) express chance constraints, the other forms' meanings are similar to the analysis of the expected value model.

\section{Hybrid intelligent algorithm}

Generally speaking, it is difficult to solve uncertain programming models. In this paper, a hybrid intelligent algorithm integrating stochastic simulation, neural network and genetic algorithm is designed to solve the above two types of stochastic models. We take the chance-constrained model as the example to introduce the hybrid intelligent algorithm.

Firstly, we apply stochastic simulations to simulate functions with random variables. The stochastic simulation is one of the most widely used techniques in stochastic system modeling, which has been applied in many fields. Although the stochastic simulation can't give the accurate results and it is also a time-consuming process, it is possibly the only effective method for complex questions which haven't analysis results(1959, p.73).

In order to solve the model, we generate training input-output data for the uncertain function $U: x \rightarrow\left(U_{1}(x), U_{2}(x), U_{3}(x)\right)$, where

$$
\begin{aligned}
& U_{1}(x)=\max \left\{\bar{f} \mid \operatorname{Pr}\left\{\sum_{i=1}^{n} k_{i} \bar{P}_{i} \geq \bar{f}\right\} \geq \alpha\right\}, \\
& U_{2}(x)=\operatorname{Pr}\left\{\sum_{i=1}^{n} \bar{P}_{i} \leq \sum_{i=1}^{n} P_{i}\right\},
\end{aligned}
$$


$U_{3}(x)=\operatorname{Pr}\left\{\sum_{i=1}^{n} \sum_{j=1}^{m} \xi_{i j} \leq \sum_{i=1}^{n} \sum_{j=1}^{m} q_{i j}\right\}$,

by the stochastic simulation. Then we train a neural network to approximate the uncertain function $U$.

Finally, the trained neural network is embedded into a genetic algorithm to produce a hybrid intelligent algorithm. The procedure can be summarized generally as follows:

Step 1. Generate training intput-output data for the above uncertain function $U$ by the stochastic simulation and then train a neural network to approximate the uncertain function $U$.

Step 2. Initialize pop_size chromosomes whose feasibility may be checked by the trained neural work.

Step 3. Update the chromosomes by crossover and mutation operations.

Step 4. Calculate the objective values for all chromosomes by the trained neural network and compute the fitness of each chromosome according to the objective values.

Step 5. Select the chromosomes by spinning the roulette wheel according to the different fitness values.

Step 6. Repeat the third to fifth steps for a given number of cycles.

Step 7. Report the best chromosome as the optimal solution of unbalanced bidding problem.

\section{Numerical experiment}

Now let us consider an unbalanced bidding problem. Assume that the client's BOQ consists of five bidding activities and each activity has six components which are man-power cost, material cost, mechanical cost, administrative charge, profit, risk cost in turn. The unit prices of man-power cost and administrative charge remain unchanged, but others change during the period of total project according to some practical cases. The budget unit prices and engineering quantities of the client for activities are presented in Table 1 and Table 2 , respectively. The monthly interest rate $r$ is given as $1 \%$.

Obviously, the budget engineering quantities are decided by the owner's careful analysis. But in the real project problem, the engineering quantities should fluctuate randomly near the owner's budget due to uncertainty and substitution of main components in each activity. So the engineering quantities must be adjusted by the contract according to the actual situation. Note that the bidding engineering quantities are assumed as random variables with uniform distributions denoted by $U(a, b)$ shown in Table 3.

\subsection{Chance-constrained model}

For example, the contractor decides to bid the project, in which the starting times and the duration times of activities are given in Table 4.

With the idea of maximizing the present value of contractor's bidding price for the total project at the predetermined confidence level subject to some chance constraints, we consider the following chance-constrained model:

$$
\left\{\begin{array}{l}
\max \bar{f} \\
\text { subject to: } \\
\operatorname{Pr}\left\{\sum_{i=1}^{5} k_{i} \overline{P_{i}} \geq \bar{f}\right\} \geq 0.95 \\
\operatorname{Pr}\left\{\sum_{i=1}^{5} \bar{P}_{i} \leq \sum_{i=1}^{5} P_{i}\right\} \geq 0.95 \\
P_{i}=\sum_{j=1}^{6} q_{i j} p_{i j}, \quad i=1,2,3,4,5 \\
\overline{P_{i}}=\sum_{j=1}^{6} \xi_{i j} x_{i j}, \quad i=1,2,3,4,5 \\
\operatorname{Pr}\left\{\sum_{i=1}^{5} \sum_{j=1}^{6} \xi_{i j} \leq \sum_{i=1}^{5} \sum_{j=1}^{6} q_{i j}\right\} \geq 0.95 \\
0.9 p_{i j} \leq x_{i j} \leq 1.1 p_{i j}, \quad i=1,2,3,4,5 ; j=1,2,3,4,5,6 .
\end{array}\right.
$$

\subsection{Model solution}

We uses Visual C++ software to realize the hybrid intelligent algorithm of stochastic chance-constrained model with the following parameters: the pop_size is 30 , the $p_{\text {_ }}$ crossover is 0.2 , the $p_{-}$mutation is 0.2 . After a run of the hybrid intelligent algorithm (5000 cycles in simulation, 1000 training data in neural network, 1000 generations in 
genetic algorithm), we obtain the optimal solution which is given in Table 5, whose objective value $\bar{f}=2738500.3$.

The result shows that the present value of bidding price for the total project is 2738500.3 Yuan when the contractor uses the unbalanced bidding strategy with stochastic bidding engineering quantities. But the the budget price for the total project is 3168000 Yuan when the owner adopts the method of linear programming. Then the present value of the budget price is 2706506.77 Yuan via conversion. In this way, the contractor can obtain surplus profit 31993.53 Yuan. The proof-test proves that this bidding strategy tallies with the actual situation completely. Therefore, the numerical result is persuasive and successful.

\section{Conclusion}

In this paper, we attempted to solve the unbalanced bidding problem with stochastic bidding engineering quantities, which is to maximze the present value of contractor's bidding price for the total project under unbalanced bidding limits and has not been studied ever before. Two types of stochastic models, including the expected value model and the chance-constrained programming model were built to meet different requirements. Then stochastic simulation, neural network and genetic algorithm were integrated to produce a hybrid intelligent algorithm to solve the numerical example. From the numerical result, we could clearly see that the hybrid intelligent algorithm could effectively solve the unbalanced bidding problem. Furthermore, this paper provided a good applied case for the practice of uncertain programming, and it also put forward a new approach for the promotion of uncertainty theory.

\section{References}

Charnes, A, \& Cooper, W. W. (1959). Chance-constrainded programming. Management Science, 6(1):73-79.

Chen, Y., Chen, J.H., \& Xia, G.M. (2005). Study on Unbalanced Bid Model Under the Figure Pattern of the Bill of Quantities. Journal of Wuhan University of Technology, 27(12):118-120.

Dayanand, N., \& Padman, R. (1997). On modeling progress payments in project networks. Journal of the Operational Research Society, 48(9):906-918.

Dayanand, N., \& Padman, R. (2001). A two stage search heuristics for scheduling payments in projects. Annals of Operation Research, 102(1):197-220.

He, Z.W., \& Xu, Y. (2007). Optimization models and solution for multi-mode project payments scheduling. Journal of Systems Engineering, 22(5):474-479.

Liu, B. (2002). Theory and Practice of Uncertain Programming. Heidelberg, Physica-Verlag.

Ulusoy, U., \& Cebelli, S. (2000). An equitable approach to the payment scheduling problem in project management. European Journal of Operational Research, 127(2):262-278.

Zhang, H.Q., Wang, F., \& Zhang, W.C. (2005). Study on bid tactics with game theory under the working model of bill of quantity. Journal of Hohai University(Natural Sciences),33(5):595-597.

Table 1. the budget unit price of the client (unit:Yuan)

\begin{tabular}{ccccccc}
\hline$p_{i j}$ & 1 & 2 & 3 & 4 & 5 & 6 \\
\hline 1 & 60 & 30 & 50 & 80 & 10 & 10 \\
2 & 60 & 15 & 15 & 80 & 5 & 5 \\
3 & 60 & 190 & 160 & 80 & 60 & 50 \\
4 & 60 & 60 & 50 & 80 & 20 & 30 \\
5 & 60 & 70 & 50 & 80 & 80 & 20 \\
\hline
\end{tabular}


Table 2. the budget engineering quantities of the client

\begin{tabular}{cccccccc}
\hline$q_{i j}$ & 1 & 2 & 3 & 4 & 5 & 6 & Sum. \\
\hline 1 & 3000 & 2000 & 3000 & 2000 & 3000 & 2000 & 15000 \\
2 & 2000 & 1200 & 1300 & 2200 & 2600 & 2700 & 12000 \\
3 & 1100 & 1000 & 1000 & 900 & 1200 & 800 & 6000 \\
4 & 10000 & 8000 & 1700 & 1500 & 4900 & 3900 & 30000 \\
5 & 200 & 200 & 100 & 350 & 500 & 450 & 1800 \\
\hline
\end{tabular}

Table 3. the bidding engineering quantities of the contractor

\begin{tabular}{ccccccc}
\hline$\xi_{i j}$ & 1 & 2 & 3 & 4 & 5 & 6 \\
\hline 1 & $U(2700,3300)$ & $U(1900,2100)$ & $U(2700,3300)$ & $U(1800,2200)$ & $U(2750,3250)$ & $U(1900,2100)$ \\
2 & $U(1800,2200)$ & $U(1150,1250)$ & $U(1200,1400)$ & $U(2000,2400)$ & $U(2400,2800)$ & $U(2600,2800)$ \\
3 & $U(1000,1200)$ & $U(950,1050)$ & $U(900,1100)$ & $U(800,1000)$ & $U(1100,1300)$ & $U(750,850)$ \\
4 & $U(8100,9900)$ & $U(8550,9450)$ & $U(1500,1900)$ & $U(1350,1650)$ & $U(4500,5300)$ & $U(3700,4100)$ \\
5 & $U(180,220)$ & $U(190,210)$ & $U(90,110)$ & $U(300,400)$ & $U(450,550)$ & $U(420,480)$ \\
\hline
\end{tabular}

Table 4. the starting times and the duration times of activities

\begin{tabular}{ccc}
\hline the $i t h$ activity & $t_{s i}$ & $t_{i}$ (month) \\
\hline 1 & Jan. 2009 & 13 \\
2 & Oct.2009 & 6 \\
3 & Nov.2009 & 5 \\
4 & Mar.2010 & 9 \\
5 & Sep.2010 & 4 \\
\hline
\end{tabular}

Table 5. the bidding unit price of the contractor (unit:Yuan)

\begin{tabular}{ccccccc}
\hline$x_{i j}$ & 1 & 2 & 3 & 4 & 5 & 6 \\
\hline 1 & 54.568 & 28.88 & 52.927 & 86.884 & 9.134 & 10.354 \\
2 & 57.337 & 14.128 & 15.406 & 86.761 & 4.693 & 4.5 \\
3 & 55.606 & 172.41 & 154.849 & 73.918 & 54.778 & 53.257 \\
4 & 64.564 & 63.98 & 52.528 & 84.835 & 19.205 & 27.633 \\
5 & 60.69 & 74.204 & 46.97 & 77.881 & 73.06 & 21.846 \\
\hline
\end{tabular}

